\title{
Full three-body problem in effective-field-theory models of gravity
}

\author{
Emmanuele Battista* \\ Dipartimento di Fisica, Complesso Universitario di Monte S. Angelo, \\ Via Cintia Edificio 6, 80126 Napoli, Italy \\ Istituto Nazionale di Fisica Nucleare, Sezione di Napoli, \\ Complesso Universitario di Monte S. Angelo, \\ Via Cintia Edificio 6, 80126 Napoli, Italy \\ Giampiero Espositd \\ Istituto Nazionale di Fisica Nucleare, Sezione di Napoli, \\ Complesso Universitario di Monte S. Angelo, \\ Via Cintia Edificio 6, 80126 Napoli, Italy
}

(Dated: June 24, 2021)

\begin{abstract}
Recent work in the literature has studied the restricted three-body problem within the framework of effective-field-theory models of gravity. This paper extends such a program by considering the full three-body problem, when the Newtonian potential is replaced by a more general central potential which depends on the mutual separations of the three bodies. The general form of the equations of motion is written down, and they are studied when the interaction potential reduces to the quantum-corrected central potential considered recently in the literature. A recursive algorithm is found for solving the associated variational equations, which describe small departures from given periodic solutions of the equations of motion. Our scheme involves repeated application of a $2 \times 2$ matrix of first-order linear differential operators.

PACS numbers: 04.60.Ds, 95.10.Ce
\end{abstract}

${ }^{*}$ E-mail: ebattista@na.infn.it

${ }^{\dagger}$ E-mail: gesposit@na.infn.it 


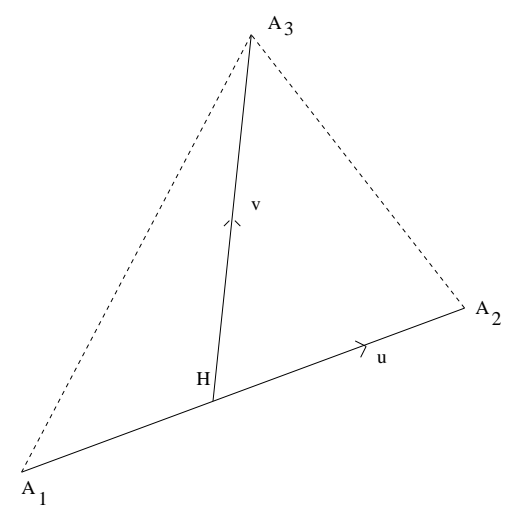

FIG. 1: The 3 bodies $A_{1}, A_{2}, A_{3}$, the center of mass $H$ of $A_{1}$ and $A_{2}$, the vector $\vec{u}$ joining $A_{1}$ to $A_{2}$, and the vector $\vec{v}$ joining $H$ to $A_{3}[12]$.

\section{INTRODUCTION}

As was stressed by Poincaré in his landmark work on the (restricted) three-body problem [1], the main aim of celestial mechanics is not the one of evaluating the astronomical ephemeris, but rather to ascertain whether Newtonian theory remains the most appropriate tool for investigating celestial gravity [2], at least (we would say) within the solar system. With hindsight, this statement is not completely superseded by current developments in gravitational theories, provided in its formulation one replaces Newtonian theory by Einstein's general relativity, which has been challenged over the years by several competing theories (e.g. Brans-Dicke, $f(R), \ldots$ ), to be tested both in the solar system and on extragalactic scales. In particular, the hybrid scheme where the Newtonian potential receives classical and quantum corrections from the calculational recipes of effective field theories has been studied in detail in Refs. [3-10] and has been applied recently to the investigation of the restricted three-body problem of celestial mechanics [11]. Interestingly, we have found that the consideration of this problem makes it possible to discriminate competing models of quantum corrections to the Newtonian potential, and that the evaluation of firstorder stability is, at least in principle, slightly affected by such tiny corrections, because the planetoid is no longer at equal distance from the two bodies of large mass, although the expected displacement from the classical equilateral triangle [12] picture is very small and not so obviously observable (see Appendix A).

It has been therefore our aim to go one step further by assessing from this novel perspective the full three-body problem of celestial mechanics. In this setting, three bodies 
$A_{1}, A_{2}, A_{3}$ having masses $m_{1}, m_{2}, m_{3}$, respectively, move in space under the action of their mutual gravitational attraction, whose functional form remains unspecified for the time being (see Secs. II and III). Following Ref. [12] we take fixed rectangular axes and denote the coordinates of $A_{r}$ at time $t$ by $x_{r}, y_{r}, z_{r}$. The coordinates of the center or mass $D$ of the three bodies are instead denoted by block capital letters $X, Y, Z$, so that, on denoting by $M \equiv m_{1}+m_{2}+m_{3}$ the mass of the whole system, one can write

$$
M X=\sum_{r=1}^{3} m_{r} x_{r}, M Y=\sum_{r=1}^{3} m_{r} y_{r}, M Z=\sum_{r=1}^{3} m_{r} z_{r} .
$$

Let the vector $\overrightarrow{A_{1} A_{2}}$ be $\vec{u}$, and let the vector $\overrightarrow{H A_{3}}$ ( $H$ being the center of mass of $A_{1}$ and $A_{2}$ ) be $\vec{v}$ (Fig. 1). Thus, by defining the parameters

$$
\alpha_{1} \equiv \frac{m_{1}}{\left(m_{1}+m_{2}\right)}, \alpha_{2} \equiv 1-\alpha_{1}
$$

the vector $\overrightarrow{A_{2} A_{3}}$ is $\left(-\alpha_{1} \vec{u}+\vec{v}\right)$, while the vector $\overrightarrow{A_{1} A_{3}}$ is $\left(\alpha_{2} \vec{u}+\vec{v}\right)$. Hereafter, we denote by $(x, y, z)$ the components of $\vec{u}$, and by $(\xi, \eta, \zeta)$ the components of $\vec{v}$. The positions and velocities of the three bodies at $t=0$ are prescribed, and the problem is to determine their position at any subsequent time.

Section II builds the Lagrangian and arrives at the general form of the equations of motion. Section III considers the choice of quantum corrected potential. Section IV writes such a general set of equations when the potential $U$ takes precisely the form considered in our previous paper [11] and suggested by the work in Refs. [3, 4, 6- 10]. Variational equations are investigated in Sec. V, and a general solution algorithm of variational equations is derived in Sec. VI. Concluding remarks and open problems are presented in Sec. VII.

\section{LAGRANGIAN AND EQUATIONS OF MOTION}

With the coordinates introduced at the end of the Introduction, the kinetic energy $T$ can be expressed by means of the relation [12]

$$
T=\frac{M}{2}\left(\dot{X}^{2}+\dot{Y}^{2}+\dot{Z}^{2}\right)+\frac{1}{2} \sum_{r<s} \frac{m_{r} m_{s}}{M} v_{r s}^{2},
$$

where $v_{r s}$ is the speed of $A_{s}$ relative to $A_{r}$, i.e.

$$
v_{r s}^{2}=\left(\dot{x}_{s}-\dot{x}_{r}\right)^{2}+\left(\dot{y}_{s}-\dot{y}_{r}\right)^{2}+\left(\dot{z}_{s}-\dot{z}_{r}\right)^{2} .
$$


On defining the "reduced masses"

$$
m \equiv \frac{m_{1} m_{2}}{\left(m_{1}+m_{2}\right)}, \mu \equiv \frac{\left(m_{1}+m_{2}\right) m_{3}}{\left(m_{1}+m_{2}+m_{3}\right)}
$$

the $x$-terms in $T$ arising from the motion relative to $D$ give [12]

$$
\frac{1}{2 M}\left[m_{2} m_{3}\left(-\alpha_{1} \dot{x}+\dot{\xi}\right)^{2}+m_{3} m_{1}\left(\alpha_{2} \dot{x}+\dot{\xi}\right)^{2}+m_{1} m_{2} \dot{x}^{2}\right]=\frac{m}{2} \dot{x}^{2}+\frac{\mu}{2} \dot{\xi}^{2} .
$$

One has now to add the corresponding formulas for $y$ and $z$, which yields the neat result

$$
T=\frac{M}{2}\left(\dot{X}^{2}+\dot{Y}^{2}+\dot{Z}^{2}\right)+\frac{m}{2}\left(\dot{x}^{2}+\dot{y}^{2}+\dot{z}^{2}\right)+\frac{\mu}{2}\left(\dot{\xi}^{2}+\dot{\eta}^{2}+\dot{\zeta}^{2}\right) .
$$

In Newtonian theory, one proceeds by assuming at this stage a potential of the form [12]

$$
U=G\left(\frac{m_{2} m_{3}}{r_{1}}+\frac{m_{3} m_{1}}{r_{2}}+\frac{m_{1} m_{2}}{r_{3}}\right)
$$

having defined

$$
\begin{gathered}
\left(r_{1}\right)^{2} \equiv\left(-\alpha_{1} \vec{u}+\vec{v}\right) \cdot\left(-\alpha_{1} \vec{u}+\vec{v}\right)=\left(-\alpha_{1} x+\xi\right)^{2}+\left(-\alpha_{1} y+\eta\right)^{2}+\left(-\alpha_{1} z+\zeta\right)^{2}, \\
\left(r_{2}\right)^{2} \equiv\left(\alpha_{2} \vec{u}+\vec{v}\right) \cdot\left(\alpha_{2} \vec{u}+\vec{v}\right)=\left(\alpha_{2} x+\xi\right)^{2}+\left(\alpha_{2} y+\eta\right)^{2}+\left(\alpha_{2} z+\zeta\right)^{2}, \\
\left(r_{3}\right)^{2} \equiv \vec{u} \cdot \vec{u}=x^{2}+y^{2}+z^{2} .
\end{gathered}
$$

In our case, although we keep using the classical concepts of kinetic energy and center of mass, we depart from classical Newtonian theory by assuming that $U$ can be a more general function of $r_{1}, r_{2}, r_{3}$, i.e.

$$
U=U\left(r_{1}, r_{2}, r_{3}\right)=\sum_{k=1}^{3} U_{k}\left(r_{k}\right) .
$$

We will first derive the equations of motion resulting from the general choice (2.10), and we will eventually look for explicit solutions with a choice of $U$ inspired by Refs. [3, 4, 10, 11].

By virtue of (2.5) and (2.10), the Lagrangian equations of motion read as

$$
M \ddot{X}=\frac{\partial U}{\partial X}, m \ddot{x}=\frac{\partial U}{\partial x}, \mu \ddot{\xi}=\frac{\partial U}{\partial \xi},
$$

supplemented by the corresponding second-order equations for $(Y, y, \eta)$ and $(Z, z, \zeta)$. Since, from (2.10), $U$ is independent of $X, Y, Z$, one has

$$
\ddot{X}=\ddot{Y}=\ddot{Z}=0,
$$


which means that the center of mass $D$ moves uniformly in a straight line. We may even assume that $D$ remains at rest without losing generality, and the remaining Eqs. for $m \ddot{x}$ and $\mu \ddot{\xi}$ in (2.11) can be obtained by setting

$$
U_{, r_{j}} \equiv \frac{\partial U}{\partial r_{j}}, \quad \forall j=1,2,3,
$$

and writing patiently the partial derivatives

$$
\begin{gathered}
\frac{\partial U}{\partial x}=U_{, r_{1}} \frac{\partial r_{1}}{\partial x}+U_{, r_{2}} \frac{\partial r_{2}}{\partial x}+U_{, r_{3}} \frac{\partial r_{3}}{\partial x}, \\
\frac{\partial U}{\partial \xi}=U_{, r_{1}} \frac{\partial r_{1}}{\partial \xi}+U_{, r_{2}} \frac{\partial r_{2}}{\partial \xi} .
\end{gathered}
$$

In light of (2.7)-(2.9), one arrives therefore at the formulas

$$
\begin{gathered}
\frac{\partial U}{\partial x}=-A x+B \xi \\
\frac{\partial U}{\partial \xi}=B x-C \xi
\end{gathered}
$$

where we have defined (cf. Eq. (29.10.11) in Ref. [12])

$$
\begin{gathered}
A \equiv-\frac{\alpha_{1}^{2}}{r_{1}} U_{, r_{1}}-\frac{\alpha_{2}^{2}}{r_{2}} U_{, r_{2}}-\frac{1}{r_{3}} U_{, r_{3}}, \\
B \equiv \frac{\alpha_{2}}{r_{2}} U_{, r_{2}}-\frac{\alpha_{1}}{r_{1}} U_{, r_{1}}, \\
C \equiv-\frac{1}{r_{1}} U_{, r_{1}}-\frac{1}{r_{2}} U_{, r_{2}} .
\end{gathered}
$$

After writing the corresponding equations for $(y, \eta)$ and $(z, \zeta)$ one obtains eventually, bearing in mind that $\vec{u}$ has components $(x, y, z)$, while $\vec{v}$ has components $(\xi, \eta, \zeta)$, the equations of motion in matrix form

$$
\left(\begin{array}{cc}
m \frac{\mathrm{d}^{2}}{\mathrm{~d} t^{2}}+A & -B \\
-B & \mu \frac{\mathrm{d}^{2}}{\mathrm{~d} t^{2}}+C
\end{array}\right)\left(\begin{array}{l}
\vec{u} \\
\vec{v}
\end{array}\right)=0 .
$$

Such a scheme tells us that the full three-body problem is equivalent to a system of two particles, i.e. a particle of mass $m$ at $(x, y, z)$ and a particle of mass $\mu$ at $(\xi, \eta, \zeta)$.

The integrals of angular momentum are found to take the form [12]

$$
\begin{aligned}
& M(Y \dot{Z}-Z \dot{Y})+m(y \dot{z}-z \dot{y})+\mu(\eta \dot{\zeta}-\zeta \dot{\eta})=a, \\
& M(Z \dot{X}-X \dot{Z})+m(z \dot{x}-x \dot{z})+\mu(\zeta \dot{\xi}-\xi \dot{\zeta})=b,
\end{aligned}
$$




$$
M(X \dot{Y}-Y \dot{X})+m(x \dot{y}-y \dot{x})+\mu(\xi \dot{\eta}-\eta \dot{\xi})=c .
$$

Since the center of mass moves uniformly in a straight line, the terms $M(Y \dot{Z}-Z \dot{Y})$ and $[m(y \dot{z}-z \dot{y})+\mu(\eta \dot{\zeta}-\zeta \dot{\eta})]$ in (2.22) are separately constant, and similarly in Eqs. (2.23) and (2.24). Indeed, one finds from Eq. (2.11)

$$
\frac{\mathrm{d}}{\mathrm{d} t}[m(y \dot{z}-z \dot{y})+\mu(\eta \dot{\zeta}-\zeta \dot{\eta})]=\left(y \frac{\partial}{\partial z}-z \frac{\partial}{\partial y}+\eta \frac{\partial}{\partial \zeta}-\zeta \frac{\partial}{\partial \eta}\right) U,
$$

which vanishes, because $U$ depends on $r_{1}, r_{2}, r_{3}$ separately, according to Eq. (2.10), and the following identity holds:

$$
\left(y \frac{\partial}{\partial z}-z \frac{\partial}{\partial y}+\eta \frac{\partial}{\partial \zeta}-\zeta \frac{\partial}{\partial \eta}\right) r_{k}=0, \forall k=1,2,3 .
$$

The forces are not in the line joining the particles, but their moment about the origin is

$$
\vec{u} \times(-A \vec{u}+B \vec{v})+\vec{v} \times(B \vec{u}-C \vec{v}),
$$

which vanishes by virtue of the skew-symmetry of the vector product. Hence the angular momentum about the origin remains constant as in Newtonian theory [12].

\section{A CHOICE OF QUANTUM CORRECTED POTENTIAL}

After having written the equations of motion in a rather general form, we cannot attempt any integration without an explicit form of the potential function. For this purpose, we now investigate the implications of assuming that the classical potential (2.6) can be replaced by a quantum corrected potential according to the recipes considered in Ref. [11]. This means that the general formula (2.10) can take the form

$$
\begin{aligned}
U\left(r_{1}, r_{2}, r_{3}\right) & =\frac{G m_{2} m_{3}}{r_{1}}\left(1+\kappa_{23} \frac{G}{c^{2}} \frac{\left(m_{2}+m_{3}\right)}{r_{1}}+\kappa \frac{l_{P}^{2}}{\left(r_{1}\right)^{2}}\right) \\
& +\frac{G m_{1} m_{3}}{r_{2}}\left(1+\kappa_{13} \frac{G}{c^{2}} \frac{\left(m_{1}+m_{3}\right)}{r_{2}}+\kappa \frac{l_{P}^{2}}{\left(r_{2}\right)^{2}}\right) \\
& +\frac{G m_{1} m_{2}}{r_{3}}\left(1+\kappa_{12} \frac{G}{c^{2}} \frac{\left(m_{1}+m_{2}\right)}{r_{3}}+\kappa \frac{l_{P}^{2}}{\left(r_{3}\right)^{2}}\right),
\end{aligned}
$$

where the parameters $\kappa, \kappa_{12}, \kappa_{23}$ and $\kappa_{13}$ are dimensionless, and $l_{P}$ is the Planck length. We stress that $\kappa_{23}, \kappa_{13}, \kappa_{12}$ depend on $\kappa$ because they are part of a calculational recipe that yields, at the same time, a post-Newtonian term and a fully quantum term. We are not 
evaluating the quantum corrections to relativistic celestial mechanics. By using Fouriertransform techniques, the $\frac{1}{q^{2}}$ term in momentum space leads to $\frac{1}{r}$, while $\frac{1}{q^{2}} \times \sqrt{q^{2}}$ and $\frac{1}{q^{2}} \times q^{2} \log \left(q^{2}\right)$ lead to $\frac{1}{r^{2}}$ and $\frac{1}{r^{3}}$, respectively. The corrections obtained in Ref. [10] result from all one-loop diagrams that can contribute to the scattering of two masses. One then finds nonanalytic corrections of the form $G m \sqrt{q^{2}}$ and $G q^{2} \log \left(q^{2}\right)$, as well as analytic terms $G q^{2}$

The first derivatives of such a potential, to be used in the definitions (2.18)-(2.20) of the functions $A, B, C$ read therefore as

$$
\begin{aligned}
& U_{, r_{1}}=-\frac{G m_{2} m_{3}}{\left(r_{1}\right)^{2}}\left(1+2 \kappa_{23} \frac{G}{c^{2}} \frac{\left(m_{2}+m_{3}\right)}{r_{1}}+3 \kappa \frac{l_{P}^{2}}{\left(r_{1}\right)^{2}}\right), \\
& U_{, r_{2}}=-\frac{G m_{1} m_{3}}{\left(r_{2}\right)^{2}}\left(1+2 \kappa_{13} \frac{G}{c^{2}} \frac{\left(m_{1}+m_{3}\right)}{r_{2}}+3 \kappa \frac{l_{P}^{2}}{\left(r_{2}\right)^{2}}\right), \\
& U_{, r_{3}}=-\frac{G m_{1} m_{2}}{\left(r_{3}\right)^{2}}\left(1+2 \kappa_{12} \frac{G}{c^{2}} \frac{\left(m_{1}+m_{2}\right)}{r_{3}}+3 \kappa \frac{l_{P}^{2}}{\left(r_{3}\right)^{2}}\right) .
\end{aligned}
$$

\section{HAMILTONIAN EQUATIONS OF MOTION}

The equations of motion (2.21) are Lagrangian second-order equations of motion. They can be re-expressed as a coupled set of twelve first-order Hamiltonian equations as follows:

$$
\begin{gathered}
\frac{\mathrm{d}}{\mathrm{d} t}=p_{x}, \\
\frac{\mathrm{d}}{\mathrm{d} t} y=p_{y}, \\
\frac{\mathrm{d}}{\mathrm{d} t}=p_{z}, \\
\frac{\mathrm{d}}{\mathrm{d} t} \xi=p_{\xi}, \\
\frac{\mathrm{d}}{\mathrm{d} t} \eta=p_{\eta}, \\
\frac{\mathrm{d}}{\mathrm{d} t} \zeta=p_{\zeta}, \\
\frac{\mathrm{d}}{\mathrm{d} t} p_{x}=-\frac{1}{m}(A x-B \xi), \\
\frac{\mathrm{d}}{\mathrm{d} t} p_{y}=-\frac{1}{m}(A y-B \eta), \\
\frac{\mathrm{d}}{\mathrm{d} t} p_{z}=-\frac{1}{m}(A z-B \zeta),
\end{gathered}
$$




$$
\begin{aligned}
\frac{\mathrm{d}}{\mathrm{d} t} p_{\xi} & =-\frac{1}{\mu}(C \xi-B x), \\
\frac{\mathrm{d}}{\mathrm{d} t} p_{\eta} & =-\frac{1}{\mu}(C \eta-B y), \\
\frac{\mathrm{d}}{\mathrm{d} t} p_{\zeta} & =-\frac{1}{\mu}(C \zeta-B z) .
\end{aligned}
$$

We need therefore twelve initial conditions to integrate these equations of motion. Hereafter it is convenient to introduce the 6 -tuple of position variables

$$
x_{i} \equiv(x, y, z, \xi, \eta, \zeta) \equiv\left(x_{1}, \ldots, x_{6}\right)
$$

and the 6-tuple of momentum variables

$$
y_{i} \equiv\left(p_{x}, p_{y}, p_{z}, p_{\xi}, p_{\eta}, p_{\zeta}\right) \equiv\left(p_{1}, \ldots, p_{6}\right)
$$

The equations (4.1)-(4.12) can be therefore further re-expressed in the canonical form [1, 2]

$$
\frac{\mathrm{d}}{\mathrm{d} t} x_{i}=\frac{\partial F}{\partial y_{i}}, \frac{\mathrm{d}}{\mathrm{d} t} y_{i}=-\frac{\partial F}{\partial x_{i}}
$$

where the function $F$ is given by

$$
F\left(x_{1}, \ldots, x_{6}, y_{1}, \ldots, y_{6}\right)=\sum_{i=1}^{6} \frac{y_{i}^{2}}{2}+f\left(x_{1}, \ldots, x_{6}\right),
$$

and $f$ solves the system of partial differential equations obtainable from (4.7)-(4.12), i.e.,

$$
\begin{aligned}
& \frac{\partial f}{\partial x}=\frac{1}{m}(A x-B \xi), \\
& \frac{\partial f}{\partial y}=\frac{1}{m}(A y-B \eta), \\
& \frac{\partial f}{\partial z}=\frac{1}{m}(A z-B \zeta), \\
& \frac{\partial f}{\partial \xi}=\frac{1}{\mu}(C \xi-B x), \\
& \frac{\partial f}{\partial \eta}=\frac{1}{\mu}(C \eta-B y), \\
& \frac{\partial f}{\partial \zeta}=\frac{1}{\mu}(C \zeta-B z),
\end{aligned}
$$

the functions $A\left(x_{1}, \ldots, x_{6}\right), B\left(x_{1}, \ldots, x_{6}\right), C\left(x_{1}, \ldots, x_{6}\right)$ being defined by (2.18)-(2.20), supplemented by (2.7)-(2.9) and (3.2)-(3.4). 
At this stage we can exploit a fundamental theorem proved by Poincaré [1, 2], according to which, if the equations (4.15), which depend on a parameter $\rho$, possess for $\rho=0 a$ periodic solution whose characteristic exponents (see Appendix) are all nonvanishing, they have again a periodic solution for small values of $\rho$. In our case, the small parameter $\rho$ is the Planck length $l_{P}$, and when $\rho=0$ we revert to the three-body problem in post-Newtonian

mechanics, for which, in the circular restricted case, one knows from recent work [13] that orbits may be unstable, or bounded chaotic, or bounded regular. In the case of Newtonian mechanics instead, Chenciner and Montgomery [14] have found a class of solutions where three bodies of equal mass move periodically on the plane along the same curve. The periodic orbit has zero angular momentum, and the three bodies chase each other around a fixed eight-shaped curve. Such an orbit visits in turn every Euler configuration in which one of the bodies sits at the midpoint of the segment defined by the other two.

To sum up, we have found that, by virtue of the Poincaré theorem on periodic solutions and of the extreme smallness of the Planck length, also our quantum corrected potential (3.1) may lead to periodic solutions. This is a novel perspective on a smooth matching between classical and quantum-corrected three-body problems.

\section{VARIATIONAL EQUATIONS}

Following Ref. [1], let us now revert to the Eqs. (4.15), and let us assume that a periodic solution has been found

$$
x_{i}=\varphi_{i}(t), y_{i}=\psi_{i}(t) .
$$

With the notation in Appendix B, we now investigate an algorithm for the evaluation of characteristic exponents. For this purpose, we consider small disturbances of such periodic solutions, written in the form

$$
\tilde{x}_{i}=\varphi_{i}(t)+\xi_{i}, \tilde{y}_{i}=\psi_{i}(t)+\eta_{i}
$$

and we form the variational equations (cf. Eq. (B4)) resulting from the linearized approximation, i.e.

$$
\begin{gathered}
\frac{\mathrm{d}}{\mathrm{d} t} \xi_{i}=\sum_{k=1}^{6}\left[F_{, y_{i} x_{k}} \xi_{k}+F_{, y_{i} y_{k}} \eta_{k}\right], \\
\frac{\mathrm{d} \eta_{i}}{\mathrm{~d} t}=-\sum_{k=1}^{6}\left[F_{, x_{i} x_{k}} \xi_{k}+F_{, x_{i} y_{k}} \eta_{k}\right],
\end{gathered}
$$


where a subscript consisting of a comma followed by a variable denotes partial derivative with respect to that variable, e.g. $F_{x_{k}} \equiv \frac{\partial F}{\partial x_{k}}$. Following Ref. [1], we try to integrate these variational equations by setting

$$
\xi_{i}=\mathrm{e}^{\alpha t} S_{i}, \eta_{i}=\mathrm{e}^{\alpha t} T_{i}
$$

$S_{i}$ and $T_{i}$ being unknown periodic functions of $t$. The work in Ref. [1] provided a remarkable proof that if, when $\rho=0$, the characteristic exponents are vanishing, then for small but nonvanishing values of $\rho$ one can expand $\alpha, S_{i}$ and $T_{i}$ in the form

$$
\begin{aligned}
\alpha & \sim \sum_{j=1}^{N} \alpha_{j} \rho^{\frac{j}{2}}, \\
S_{i} & \sim \sum_{l=0}^{N} S_{i}^{l} \rho^{\frac{l}{2}}, \\
T_{i} & \sim \sum_{l=0}^{N} T_{i}^{l} \rho^{\frac{l}{2}} .
\end{aligned}
$$

This framework is complementary to the one mentioned at the end of Sec. IV, where we mentioned the Poincaré theorem on the persistence of periodic solutions at small $\rho$. That theorem assumes instead that, at $\rho=0$, the characteristic exponents $\alpha$ are all nonvanishing.

We now insert the formulas (5.5)-(5.8) into the variational equations (5.3) and (5.4), assuming for $F$ the asymptotic expansion (in Sec. VI we will see that $F_{1}$ and $\mathrm{O}\left(\rho^{3}\right)$ vanish identically in our model)

$$
F \sim F_{0}+\rho F_{1}+\rho^{2} F_{2}+\mathrm{O}\left(\rho^{3}\right)
$$

Now the asymptotic expansion of left-hand side of the variational equations (5.3) and (5.4) yields

$$
\begin{aligned}
& \frac{\mathrm{d}}{\mathrm{d} t} \xi_{i} \sim \mathrm{e}^{\alpha t}\left[\frac{\mathrm{d} S_{i}^{0}}{\mathrm{~d} t}+\left(\alpha_{1} S_{i}^{0}+\frac{\mathrm{d} S_{i}^{1}}{\mathrm{~d} t}\right) \sqrt{\rho}+\left(\alpha_{1} S_{i}^{1}+\alpha_{2} S_{i}^{0}+\frac{\mathrm{d} S_{i}^{2}}{\mathrm{~d} t}\right) \rho+\mathrm{O}\left(\rho^{\frac{3}{2}}\right)\right], \\
& \frac{\mathrm{d}}{\mathrm{d} t} \eta_{i} \sim \mathrm{e}^{\alpha t}\left[\frac{\mathrm{d} T_{i}^{0}}{\mathrm{~d} t}+\left(\alpha_{1} T_{i}^{0}+\frac{\mathrm{d} T_{i}^{1}}{\mathrm{~d} t}\right) \sqrt{\rho}+\left(\alpha_{1} T_{i}^{1}+\alpha_{2} T_{i}^{0}+\frac{\mathrm{d} T_{i}^{2}}{\mathrm{~d} t}\right) \rho+\mathrm{O}\left(\rho^{\frac{3}{2}}\right)\right],
\end{aligned}
$$

so that comparison of coefficients of equal powers of $\rho$ yields for all $i=1, \ldots, 6$, up to first order in $\rho$, the equations

$$
\frac{\mathrm{d} S_{i}^{0}}{\mathrm{~d} t}=\sum_{k=1}^{6}\left(F_{0, y_{i} x_{k}} S_{k}^{0}+F_{0, y_{i} y_{k}} T_{k}^{0}\right)
$$




$$
\begin{gathered}
\alpha_{1} S_{i}^{0}+\frac{\mathrm{d} S_{i}^{1}}{\mathrm{~d} t}=\sum_{k=1}^{6}\left(F_{0, y_{i} x_{k}} S_{k}^{1}+F_{0, y_{i} y_{k}} T_{k}^{1}\right) \\
\alpha_{1} S_{i}^{1}+\alpha_{2} S_{i}^{0}+\frac{\mathrm{d} S_{i}^{2}}{\mathrm{~d} t}=\sum_{k=1}^{6}\left(F_{0, y_{i} x_{k}} S_{k}^{2}+F_{1, y_{i} x_{k}} S_{k}^{0}+F_{0, y_{i} y_{k}} T_{k}^{2}+F_{1, y_{i} y_{k}} T_{k}^{0}\right) \\
\frac{\mathrm{d} T_{i}^{0}}{\mathrm{~d} t}=-\sum_{k=1}^{6}\left(F_{0, x_{i} x_{k}} S_{k}^{0}+F_{0, x_{i} y_{k}} T_{k}^{0}\right) \\
\alpha_{1} T_{i}^{0}+\frac{\mathrm{d} T_{i}^{1}}{\mathrm{~d} t}=-\sum_{k=1}^{6}\left(F_{0, x_{i} x_{k}} S_{k}^{1}+F_{0, x_{i} y_{k}} T_{k}^{1}\right) \\
\alpha_{1} T_{i}^{1}+\alpha_{2} T_{i}^{0}+\frac{\mathrm{d} T_{i}^{2}}{\mathrm{~d} t}=-\sum_{k=1}^{6}\left(F_{0, x_{i} x_{k}} S_{k}^{2}+F_{1, x_{i} x_{k}} S_{k}^{0}+F_{0, x_{i} y_{k}} T_{k}^{2}+F_{1, x_{i} y_{k}} T_{k}^{0}\right)
\end{gathered}
$$

To begin, one should solve Eqs. (5.12) and (5.15) for $S_{i}^{0}$ and $T_{i}^{0}$, and insert them into (5.13) and (5.16) to find $S_{i}^{1}$ and $T_{i}^{1}$, and iterate the procedure to find $S_{i}^{2}, T_{i}^{2}, \ldots$ as well as $\alpha_{1}, \alpha_{2}, \ldots$

\section{GENERAL SOLUTION OF VARIATIONAL EQUATIONS}

For the purpose of finding a general solution of variational equations, it may be helpful to elaborate the equations of Sec. IV, where the potential term $U$ of Eq. (3.1) contains only a part of zeroth-order in $\rho \equiv l_{P}$ and a part of second order in $\rho$, and the same holds for the Hamiltonian function $F$ in (4.16). More precisely, on defining

$$
\begin{aligned}
& \gamma_{1}\left(r_{1}\right) \equiv-\frac{G m_{2} m_{3}}{\left(r_{1}\right)^{2}}\left(1+2 \kappa_{23} \frac{G}{c^{2}} \frac{\left(m_{2}+m_{3}\right)}{r_{1}}\right) \\
& \gamma_{2}\left(r_{2}\right) \equiv-\frac{G m_{1} m_{3}}{\left(r_{2}\right)^{2}}\left(1+2 \kappa_{13} \frac{G}{c^{2}} \frac{\left(m_{1}+m_{3}\right)}{r_{2}}\right) \\
& \gamma_{3}\left(r_{3}\right) \equiv-\frac{G m_{1} m_{2}}{\left(r_{3}\right)^{2}}\left(1+2 \kappa_{12} \frac{G}{c^{2}} \frac{\left(m_{1}+m_{2}\right)}{r_{3}}\right),
\end{aligned}
$$

we find that $A, B$ and $C$ in (2.18)-(2.20) take the form

$$
A=A_{0}+\rho^{2} A_{2}, B=B_{0}+\rho^{2} B_{2}, C=C_{0}+\rho^{2} C_{2},
$$

where

$$
\begin{gathered}
A_{0}=-\left(\alpha_{1}\right)^{2} \frac{\gamma_{1}\left(r_{1}\right)}{r_{1}}-\left(\alpha_{2}\right)^{2} \frac{\gamma_{2}\left(r_{2}\right)}{r_{2}}-\frac{\gamma_{3}\left(r_{3}\right)}{r_{3}}, \\
A_{2}=3 G \kappa\left[\left(\alpha_{1}\right)^{2} \frac{m_{2} m_{3}}{\left(r_{1}\right)^{5}}+\left(\alpha_{2}\right)^{2} \frac{m_{1} m_{3}}{\left(r_{2}\right)^{5}}+\frac{m_{1} m_{2}}{\left(r_{3}\right)^{5}}\right],
\end{gathered}
$$




$$
\begin{gathered}
B_{0}=\alpha_{2} \frac{\gamma_{2}\left(r_{2}\right)}{r_{2}}-\alpha_{1} \frac{\gamma_{1}\left(r_{1}\right)}{r_{1}}, \\
B_{2}=3 G \kappa\left[\alpha_{1} \frac{m_{2} m_{3}}{\left(r_{1}\right)^{5}}-\alpha_{2} \frac{m_{1} m_{3}}{\left(r_{2}\right)^{5}}\right], \\
C_{0}=-\frac{\gamma_{1}\left(r_{1}\right)}{r_{1}}-\frac{\gamma_{2}\left(r_{2}\right)}{r_{2}}, \\
C_{2}=3 G \kappa\left[\frac{m_{1} m_{3}}{\left(r_{2}\right)^{5}}+\frac{m_{2} m_{3}}{\left(r_{1}\right)^{5}}\right] .
\end{gathered}
$$

At this stage, the coupled system (4.17)-(4.22) can be re-expressed in the form

$$
\begin{aligned}
\frac{\partial f}{\partial x_{1}} & =\frac{1}{m}\left(A_{0} x_{1}-B_{0} x_{4}\right)+\frac{1}{m}\left(A_{2} x_{1}-B_{2} x_{4}\right) \rho^{2}, \\
\frac{\partial f}{\partial x_{2}} & =\frac{1}{m}\left(A_{0} x_{2}-B_{0} x_{5}\right)+\frac{1}{m}\left(A_{2} x_{2}-B_{2} x_{5}\right) \rho^{2}, \\
\frac{\partial f}{\partial x_{3}} & =\frac{1}{m}\left(A_{0} x_{3}-B_{0} x_{6}\right)+\frac{1}{m}\left(A_{2} x_{3}-B_{2} x_{6}\right) \rho^{2}, \\
\frac{\partial f}{\partial x_{4}} & =\frac{1}{\mu}\left(C_{0} x_{4}-B_{0} x_{1}\right)+\frac{1}{\mu}\left(C_{2} x_{4}-B_{2} x_{1}\right) \rho^{2}, \\
\frac{\partial f}{\partial x_{5}} & =\frac{1}{\mu}\left(C_{0} x_{5}-B_{0} x_{2}\right)+\frac{1}{\mu}\left(C_{2} x_{5}-B_{2} x_{2}\right) \rho^{2}, \\
\frac{\partial f}{\partial x_{6}} & =\frac{1}{\mu}\left(C_{0} x_{6}-B_{0} x_{3}\right)+\frac{1}{\mu}\left(C_{2} x_{6}-B_{2} x_{3}\right) \rho^{2},
\end{aligned}
$$

where the left-hand sides can be further re-expressed upon writing

$$
f\left(x_{1}, \ldots, x_{6}\right)=f_{0}\left(x_{1}, \ldots, x_{6}\right)+f_{2}\left(x_{1}, \ldots, x_{6}\right) \rho^{2} .
$$

On the one hand, from (4.16) and (6.17) we have immediately that

$$
F_{0, x_{i} y_{k}}=f_{0, x_{i} y_{k}}=0, F_{0, y_{i} x_{k}}=\left(y_{i}\right)_{, x_{k}}=0, F_{0, y_{i} y_{k}}=\delta_{i k}, F_{0, x_{i} x_{k}}=f_{0, x_{i} x_{k}} .
$$

On the other hand, from Eqs. (6.11)-(6.17), we find immediately the $6 \times 6$ matrix of partial derivatives

$$
M_{i k}^{0} \equiv f_{0, x_{i} x_{k}}
$$

whose entries are written explicitly, for completeness, in Appendix C. Now a patient application of (4.16), (6.17) and (6.18) to the Eqs. (5.12)-(5.17) reveals that, for all $i=1, \ldots, 6$ (exploiting the vanishing of $F_{1}$ in our model)

$$
\sum_{k=1}^{6}\left(\begin{array}{cc}
\delta_{i k} \frac{\mathrm{d}}{\mathrm{d} t} & -\delta_{i k} \\
M_{i k}^{0} & \delta_{i k} \frac{\mathrm{d}}{\mathrm{d} t}
\end{array}\right)\left(\begin{array}{c}
S_{k}^{0} \\
T_{k}^{0}
\end{array}\right)=0
$$


while, for higher-order terms, we find the inhomogeneous equations

$$
\sum_{k=1}^{6}\left(\begin{array}{cc}
\delta_{i k} \frac{\mathrm{d}}{\mathrm{d} t} & -\delta_{i k} \\
M_{i k}^{0} & \delta_{i k} \frac{\mathrm{d}}{\mathrm{d} t}
\end{array}\right)\left(\begin{array}{c}
S_{k}^{n} \\
T_{k}^{n}
\end{array}\right)=-\sum_{l=0}^{n-1} \alpha_{n-l}\left(\begin{array}{c}
S_{i}^{l} \\
T_{i}^{l}
\end{array}\right)
$$

For example, for the equations involving $\alpha_{1}$ and $\alpha_{2}$ we find

$$
\begin{gathered}
\sum_{k=1}^{6}\left(\begin{array}{cc}
\delta_{i k} \frac{\mathrm{d}}{\mathrm{d} t} & -\delta_{i k} \\
M_{i k}^{0} & \delta_{i k} \frac{\mathrm{d}}{\mathrm{d} t}
\end{array}\right)\left(\begin{array}{c}
S_{k}^{1} \\
T_{k}^{1}
\end{array}\right)=-\alpha_{1}\left(\begin{array}{c}
S_{i}^{0} \\
T_{i}^{0}
\end{array}\right), \\
\sum_{k=1}^{6}\left(\begin{array}{cc}
\delta_{i k} \frac{\mathrm{d}}{\mathrm{d} t} & -\delta_{i k} \\
M_{i k}^{0} & \delta_{i k} \frac{\mathrm{d}}{\mathrm{d} t}
\end{array}\right)\left(\begin{array}{c}
S_{k}^{2} \\
T_{k}^{2}
\end{array}\right)=-\alpha_{2}\left(\begin{array}{c}
S_{i}^{0} \\
T_{i}^{0}
\end{array}\right)-\alpha_{1}\left(\begin{array}{c}
S_{i}^{1} \\
T_{i}^{1}
\end{array}\right) .
\end{gathered}
$$

\section{A. The case when $\alpha$ does not vanish at $\rho=0$}

As we know from Sec. IV, it is at least equally important to study the case when the characteristic exponent does not vanish at $\rho=0$ [1, 2]. In such a case, we assume that the asymptotic expansion (5.6) can be generalized by adding the term $\alpha_{0}$, i.e.

$$
\alpha \sim \sum_{l=0}^{N} \alpha_{l} \rho^{\frac{l}{2}}
$$

The method of Secs. V and VI leads eventually to equations that generalize (6.20)-(6.23) upon adding $\alpha_{0}$ to the linear differential operator $\frac{\mathrm{d}}{\mathrm{d} t}$, i.e.

$$
\begin{gathered}
\sum_{k=1}^{6}\left(\begin{array}{cc}
\delta_{i k}\left(\frac{\mathrm{d}}{\mathrm{d} t}+\alpha_{0}\right) & -\delta_{i k} \\
M_{i k}^{0} & \delta_{i k}\left(\frac{\mathrm{d}}{\mathrm{d} t}+\alpha_{0}\right)
\end{array}\right)\left(\begin{array}{c}
S_{k}^{0} \\
T_{k}^{0}
\end{array}\right)=0 \\
\sum_{k=1}^{6}\left(\begin{array}{cc}
\delta_{i k}\left(\frac{\mathrm{d}}{\mathrm{d} t}+\alpha_{0}\right) & -\delta_{i k} \\
M_{i k}^{0} & \delta_{i k}\left(\frac{\mathrm{d}}{\mathrm{d} t}+\alpha_{0}\right)
\end{array}\right)\left(\begin{array}{c}
S_{k}^{n} \\
T_{k}^{n}
\end{array}\right)=-\sum_{l=0}^{n-1} \alpha_{n-l}\left(\begin{array}{c}
S_{i}^{l} \\
T_{i}^{l}
\end{array}\right) .
\end{gathered}
$$

\section{B. Hamiltonian equations when $\rho=0$}

Our computational recipes are of little help unless we say what sort of periodic solutions we have in mind. Since we are interested in small departures from classical theory, and $\rho \equiv l_{P}$ is the naturally occurring parameter to describe such a scheme, we assume hereafter that the periodic solutions alluded to in Eq. (5.1) are solutions of Eqs. (4.15) when $\rho=0$. 
With the notation in Eqs. (6.1)-(6.3), (6.5), (6.7) and (6.9), the matrix (6.19) should be therefore evaluated along solutions of the coupled equations

$$
\begin{gathered}
\frac{\mathrm{d} x_{i}}{\mathrm{~d} t}=y_{i} \forall i=1, \ldots, 6, \\
\frac{\mathrm{d} y_{i}}{\mathrm{~d} t}=-\frac{1}{m}\left(A_{0} x_{i}-B_{0} x_{i+3}\right) \forall i=1,2,3, \\
\frac{\mathrm{d} y_{i}}{\mathrm{~d} t}=-\frac{1}{\mu}\left(C_{0} x_{i}-B_{0} x_{i-3}\right) \forall i=4,5,6 .
\end{gathered}
$$

The desired periodic solutions, whose existence is a special rather than generic property [1, 2, 13], can be written in the form

$$
\begin{aligned}
& x_{i}=\sum_{l=0}^{\infty} D_{i l} \sin \left(\omega_{i l} t+\varphi_{i l}\right), \\
& y_{i}=\sum_{l=0}^{\infty} E_{i l} \sin \left(\omega_{i l} t+\gamma_{i l}\right) .
\end{aligned}
$$

When we insert such Fourier expansions into the system (6.27)-(6.29) we have to bear in mind that $A_{0}, B_{0}, C_{0}$ in $(6.5),(6.7),(6.9)$ depend on $x_{1}, \ldots, x_{6}$ because Eqs. (2.7)-(2.9) can be re-expressed in the form

$$
\left(r_{1}\right)^{2}=\sum_{k=1}^{3}\left(\alpha_{1} x_{k}-x_{k+3}\right)^{2},\left(r_{2}\right)^{2}=\sum_{k=1}^{3}\left(\alpha_{2} x_{k}+x_{k+3}\right)^{2},\left(r_{3}\right)^{2}=\sum_{k=1}^{3}\left(x_{k}\right)^{2} .
$$

\section{CONCLUDING REMARKS AND OPEN PROBLEMS}

The equations of Sec. VI for the evaluation of solutions of the variational equations of Sec. V are our main original result. We have arrived at a broad framework that presents formidable technical difficulties, which is not the same as solving our equations. For this purpose, one should solve completely the following problems:

(i) First, how to find periodic solutions of the Hamiltonian equations (4.15) when $\rho=0$. From Eqs. (6.27)-(6.31), this means having to solve the infinite system of equations

$$
\begin{aligned}
\sum_{l=0}^{\infty} D_{i l} \omega_{i l} \cos \left(\omega_{i l} t\right. & \left.+\varphi_{i l}\right)=\sum_{l=0}^{\infty} E_{i l} \sin \left(\omega_{i l} t+\gamma_{i l}\right), \forall i=1, \ldots, 6 \\
\sum_{l=0}^{\infty} E_{i l} \omega_{i l} \cos \left(\omega_{i l} t+\gamma_{i l}\right) & =-\frac{A_{0}}{m} \sum_{l=0}^{\infty} D_{i l} \sin \left(\omega_{i l} t+\gamma_{i l}\right) \\
& +\frac{B_{0}}{m} \sum_{l=0}^{\infty} D_{i+3, l} \sin \left(\omega_{i+3, l} t+\gamma_{i+3, l}\right), \forall i=1,2,3
\end{aligned}
$$




$$
\begin{aligned}
\sum_{l=0}^{\infty} E_{i l} \omega_{i l} \cos \left(\omega_{i l} t+\gamma_{i l}\right) & =-\frac{C_{0}}{\mu} \sum_{l=0}^{\infty} D_{i l} \sin \left(\omega_{i l} t+\gamma_{i l}\right) \\
& +\frac{B_{0}}{\mu} \sum_{l=0}^{\infty} D_{i-3, l} \sin \left(\omega_{i-3, l} t+\gamma_{i-3, l}\right), \forall i=4,5,6 .
\end{aligned}
$$

(ii) Second, how to solve the variational equations through Eqs. (6.20) and (6.21), or (6.25) and (6.26), when the matrix $M_{i k}^{0}$ is evaluated along a solution of Eqs. (7.1)-(7.3). In Refs. [1, 2], Poincaré obtained an algebraic equation of third degree for the square of $\alpha_{1}$, which was the hardest part of the calculation, but we do not see an analogous equation for the square of $\alpha_{1}$ in our case.

(iii) Third, what is the counterpart, if any, of the variety of periodic and asymptotic solutions found by Poincaré [1, 2], i.e., more precisely:

(iii-a) Periodic solutions of the Hamiltonian equations (4.15) with nonvanishing values of $\rho$, e.g.

$$
x_{l}(t)=\psi_{l}^{0}(t)+\left(\rho-\rho_{0}\right)^{\frac{1}{2}} \psi_{l}^{(1)}(t)+\left(\rho-\rho_{0}\right) \psi_{l}^{(2)}(t)+\left(\rho-\rho_{0}\right)^{\frac{3}{2}} \psi_{l}^{(3)}(t)+\ldots,
$$

where $\psi_{l}^{0}(t)$ has period $T$, while $\psi_{l}^{(1)}(t), \psi_{l}^{(2)}(t), \psi_{l}^{(3)}(t)$ have period equal to an integer multiple of $T$.

(iii-b) Asymptotic solutions of Eqs. (4.15) of the first kind, for which

$$
x_{i}(t)=\varphi_{i}(t)+A \mathrm{e}^{-\alpha t} \theta_{i}^{(1)}(t)+A^{2} \mathrm{e}^{-2 \alpha t} \theta_{i}^{(2)}(t)+A^{3} \mathrm{e}^{-3 \alpha t} \theta_{i}^{(3)}(t)+\ldots
$$

where $\varphi_{i}(t)$ is an unstable periodic solution, $A$ is an arbitrary integration constant, $\alpha$ is a positive characteristic exponent, $\theta_{i}^{(1)}(t), \theta_{i}^{(2)}(t) \ldots$ have period $T$. At sufficiently large positive values of $t$ such series are convergent. As $t \rightarrow \infty$, such solutions approach asymptotically the unstable periodic solution $\varphi_{i}(t)$.

(iii-c) Asymptotic solutions of Eqs. (4.15) of the second kind, for which

$$
x_{i}(t)=\varphi_{i}(t)+B \mathrm{e}^{\alpha t} \omega_{i}^{(1)}(t)+B^{2} \mathrm{e}^{2 \alpha t} \omega_{i}^{(2)}(t)+B^{3} \mathrm{e}^{3 \alpha t} \omega_{i}^{(3)}(t)+\ldots
$$

where $B$ is a new integration constant, $\alpha$ is again the positive characteristic exponent, and the functions $\omega$ are of the same functional form as the functions $\theta$ occurring in (7.5). At sufficiently large negative values of $t$ such series are convergent. As $t \rightarrow-\infty$, such solutions approach asymptotically the unstable periodic solution $\varphi_{i}(t)$. 
(iii-d) Doubly asymptotic solutions which are represented by (7.6) if $t<0$ and $|t|$ is very large, and by (7.5) if $t>0$ and $|t|$ is very large. The corresponding orbit, which initially differs slightly from the unstable periodic solution, departs gradually from it at first, and after having departed significantly from it ends up by approaching asymptotically the unstable periodic solution. At finite values of $t$, there exist intervals of this time variable where neither (7.5) nor (7.6) converges in Newtonian physics [1, 2].

One should notice that the actual evaluation of periodic solutions of the full three-body problem within the framework of parametrized post-Newtonian formalism is still in its infancy, since, to the best of our knowledge, only results for the circular restricted three-body problem are available so far [13], unlike the case of Newtonian theory, where, after centuries of efforts, some periodic solutions of the full three-body problem are explicitly known by now [14]. The years to come will hopefully tell us whether the scheme described by our Sec. VI may have observational consequences (see also the numerical estimates in Appendix A) in orbital motion physics and in the experimental search for quantum gravity effects (see below).

A naturally occurring question is to what extent is it legitimate to keep using the Lagrangian and Hamiltonian frameworks of classical mechanics, jointly with its set of variational equations, once the quantum corrections of Refs. [3, 5, 10] have been obtained. As far as we can see, a possible answer is as follows. The work of Refs. [3, 5, 10] deals with the leading long distance quantum corrections to the Newtonian potential, and leads, by construction, to low-energy effects, here considered in the solar system. These result entirely from the Einstein-Hilbert part of the full Lagrangian of gravity. The high-energy effects are instead ruled by terms of higher order in the curvature, e.g.

$$
R_{\alpha \beta \gamma \delta} R^{\alpha \beta \gamma \delta}, R_{\alpha \beta} R^{\alpha \beta}, R^{2}, \square R, R_{\alpha \beta}^{\lambda \mu} R_{\lambda \mu}^{\nu \rho} R_{\nu \rho}^{\alpha \beta}
$$

in the quantum effective action (the generating functional of one-particle irreducible diagrams), which lead however to quantum corrections at long distances severely suppressed with respect to the ones considered in the papers [3, 5, 10]. Of course, our scheme belongs to the family of hybrid schemes in theoretical physics, discussed in detail in the Introduction of our previous paper [11].

Furthermore, the use of classical mechanics is suggested by the very nature of the quantum corrections obtained in Refs. [3, 5, 10]: a post-Newtonian term, and a quantum term 
containing a very small parameter, i.e. the square of Planck length.

Last, but not least, our predictions have chances of being testable against observations. In the Earth-Moon-satellite system, we find, with the notation in appendix A, that the planetoid (i.e. satellite) coordinates at the Lagrangian points of stable equilibrium are

$$
x_{Q}=1.8732985853448734 \cdot 10^{8} \mathrm{~m}, y_{Q}= \pm 3.3255375505843085 \cdot 10^{8} \mathrm{~m},
$$

whereas the classical Newtonian values are

$$
x_{C}=1.8732985852568874 \cdot 10^{8} \mathrm{~m}, y_{C}= \pm 3.3255375505322444 \cdot 10^{8} \mathrm{~m} .
$$

This means that we predict a quantum correction to the $x$ coordinate given by

$$
x_{Q}-x_{C} \approx 0.879 \mathrm{~cm},
$$

while

$$
\left|y_{Q}\right|-\left|y_{C}\right| \approx 0.52 \mathrm{~cm} \text {. }
$$

Interestingly, these corrections are within reach of current technology, and we arrive at a prediction of low-energy quantum gravity effects in the solar system, which was, to our knowledge, quite unexpected. In the near future we hope to be able to propose measurements aimed at testing such an effect, which can receive careful consideration, in light of the broad interest of the scientific community in the applications of Lagrangian points in the solar system [15 17].

\section{Acknowledgments}

The authors are indebted to John Donoghue for enlightening correspondence, and to Massimo Cerdonio and Alberto Vecchiato for conversations. G. E. is grateful to the Dipartimento di Fisica of Federico II University, Naples, for hospitality and support.

Appendix A: Closer look at stable equilibrium points of the restricted three-body problem

In Ref. [11], with the notation described therein, according to which the planetoid is at distance $r$ from the body $A$ of mass $\alpha$ and coordinates $(-a, 0)$, and at distance $s$ from the 
body $B$ of mass $\beta$ and coordinates $(b, 0)$, the coordinates of stable equilibrium points of the planetoid are (hereafter $l \equiv(a+b))$

$$
\begin{gathered}
x(l)=\frac{\left(r^{2}(l)-s^{2}(l)+b^{2}-a^{2}\right)}{2(a+b)}, \\
y_{ \pm}(l)= \pm \sqrt{r^{2}(l)-x^{2}(l)-2 a x(l)-a^{2}},
\end{gathered}
$$

where

$$
r(l)=\frac{1}{w_{+}(l)}, s(l)=\frac{1}{u_{+}(l)},
$$

$w_{+}$and $u_{+}$being the positive roots of the algebraic equation of fifth degree

$$
\sum_{k=0}^{5} \zeta_{k} w^{k}=0, \sum_{k=0}^{5} \tilde{\zeta}_{k} u^{k}=0
$$

where

$$
\begin{gathered}
\zeta_{5}=1, \zeta_{4}=\frac{2}{3} \frac{\kappa_{1}}{\kappa_{2}} \frac{G(m+\alpha)}{c^{2} l_{P}^{2}}, \zeta_{3}=\frac{1}{3 \kappa_{2}} \frac{1}{l_{P}^{2}}, \\
\zeta_{2}=\zeta_{1}=0, \zeta_{0}=-\frac{1}{3 \kappa_{2}} \frac{1}{l_{P}^{2} l^{3}}, \\
\tilde{\zeta}_{k}=\zeta_{k} \forall k=0,1,2,3,5, \tilde{\zeta}_{4}=\frac{2}{3} \frac{\kappa_{3}}{\kappa_{2}} \frac{G(m+\beta)}{c^{2} l_{P}^{2}} .
\end{gathered}
$$

In Ref. [11] we have solved numerically such algebraic equations, since no general algorithm exists for solving algebraic equations of fifth or higher degree. However, since the left-hand side of Eqs. (A4) is a fairly simple polynomial function, the basic rules for studying functions of a real variable provide already a valuable information. For example, one has

$$
f^{\prime}(w)=w^{2}\left(3 \zeta_{3}+4 \zeta_{4} w+5 w^{2}\right)
$$

which therefore vanishes either at $w=0$ or at

$$
\begin{aligned}
& w_{1}=-\frac{2}{5} \zeta_{4}+\frac{1}{5} \sqrt{4 \zeta_{4}^{2}-15 \zeta_{3}^{2}}, \\
& w_{2}=-\frac{2}{5} \zeta_{4}-\frac{1}{5} \sqrt{4 \zeta_{4}^{2}-15 \zeta_{3}^{2}} .
\end{aligned}
$$

By virtue of (A5), such roots are real provided that

$$
\frac{\left(\kappa_{1}\right)^{2}}{\kappa_{2}}>\frac{45}{16} \frac{c^{4}}{G^{2}(m+\alpha)} l_{P}^{2},
$$


which is satisfied in the Sun-Earth-Moon and Jupiter-Ganimede-Adrastea systems by virtue of the small value of the Planck length. The roots $w_{1}$ and $w_{2}$ are therefore both negative. Moreover, the second derivative of $f$ reads as

$$
f^{\prime \prime}(w)=2 w\left(3 \zeta_{3}+6 \zeta_{4} w+10 w^{2}\right) \equiv 2 w g(w) .
$$

The point $w=0$ is therefore a flex point, while the sign of $f^{\prime \prime}$ at $w_{1}$ and $w_{2}$, and hence maxima or minima of $f$, is governed by the sign of the second degree polynomial $g(w) \equiv$ $3 \zeta_{3}+6 \zeta_{4} w+10 w^{2}$.

Interestingly, from Eqs. (A1) and (A2) we find for the Sun-Earth-Moon system the quantum corrected planetoid (i.e. the Moon) coordinates at equilibrium

$$
x_{Q}=7,479978 \cdot 10^{10} \mathrm{~m}, y_{Q}=1,29573 \cdot 10^{11} \mathrm{~m},
$$

to be compared with the classical Newtonian values

$$
x_{C}=7,479955 \cdot 10^{10} \mathrm{~m}, y_{C}=1,29557 \cdot 10^{11} \mathrm{~m} .
$$

Moreover, for the Jupiter-Ganimede-Adrastea system, we find the quantum corrected planetoid coordinates (i.e. Adrastea) at equilibrium

$$
x_{Q}=5,349183 \cdot 10^{8} \mathrm{~m}, y_{Q}=9,2698 \cdot 10^{8} \mathrm{~m},
$$

whereas the classical Newtonian values are

$$
x_{C}=5,349167 \cdot 10^{8} \mathrm{~m}, y_{C}=9,2665 \cdot 10^{8} \mathrm{~m} .
$$

As one can see, in both cases, the $x$-values start differing at the fifth decimal digit, while the $y$-values may start differing at the fourth or third decimal digit.

\section{Appendix B: Definition of characteristic exponents}

Following Refs. [1, 2], consider the differential equations

$$
\frac{\mathrm{d}}{\mathrm{d} t} x_{i}=X_{i},
$$

and suppose they admit a periodic solution

$$
x_{i}=\varphi_{i}(t) .
$$


We can now consider small disturbances of Eqs. (B1) by setting

$$
x_{i}=\varphi_{i}(t)+\xi_{i}
$$

and neglecting the squares of the $\xi_{i}$. We are therefore studying the linearized perturbative regime for Eqs. (B1). This procedure leads to the first-order equations

$$
\frac{\mathrm{d}}{\mathrm{d} t} \xi_{i}=\sum_{k=1}^{n} \frac{\partial X_{i}}{\partial x_{k}} \xi_{k}
$$

known as the variational equations [12]. These equations are linear with respect to the $\xi_{k}$, and their coefficients $\frac{\partial X_{i}}{\partial x_{k}}$, where $x_{i}$ should be eventually replaced by $\varphi_{i}(t)$, are periodic functions of the time variable $t$. Hence we have to integrate linear differential equations with periodic coefficients. The general form of the solutions of these equations has been known for centuries; one obtains $n$ particular solutions of the following form:

$$
\xi_{1}=\mathrm{e}^{\alpha_{k} t} S_{1 k}, \xi_{2}=\mathrm{e}^{\alpha_{k} t} S_{2 k}, \ldots, \xi_{n}=\mathrm{e}^{\alpha_{k} t} S_{n k},
$$

for all $k=1,2, \ldots, n$, the $\alpha_{k}$ being constants and the $S_{i k}$ being periodic functions of $t$ with the same period as the $\varphi_{i}(t)$. The constants $\alpha_{k}$ are said to be the characteristic exponents

of the periodic solutions [1, 2]. Our equations of motion (4.15) belong to the general family expressed by (B1).

\section{Appendix C: The matrix $M_{i k}^{0}$}

For the matrix of partial derivatives defined in Eq. (6.19) we find (with the understanding that a subscript like ${ }_{, k}$ denotes partial derivative with respect to $x_{k}$, for all $k=1, \ldots, 6$ )

$$
\begin{gathered}
M_{11}^{0}=\frac{1}{m}\left(x_{1} A_{0,1}+A_{0}-x_{4} B_{0,1}\right), \\
M_{12}^{0}=\frac{1}{m}\left(x_{1} A_{0,2}-x_{4} B_{0,2}\right), \\
M_{13}^{0}=\frac{1}{m}\left(x_{1} A_{0,3}-x_{4} B_{0,3}\right), \\
M_{14}^{0}=\frac{1}{m}\left(x_{1} A_{0,4}-x_{4} B_{0,4}-B_{0}\right), \\
M_{15}^{0}=\frac{1}{m}\left(x_{1} A_{0,5}-x_{4} B_{0,5}\right),
\end{gathered}
$$




$$
\begin{aligned}
& M_{16}^{0}=\frac{1}{m}\left(x_{1} A_{0,6}-x_{4} B_{0,6}\right), \\
& M_{21}^{0}=\frac{1}{m}\left(x_{2} A_{0,1}-x_{5} B_{0,1}\right), \\
& M_{22}^{0}=\frac{1}{m}\left(x_{2} A_{0,2}+A_{0}-x_{5} B_{0,2}\right) \text {, } \\
& M_{23}^{0}=\frac{1}{m}\left(x_{2} A_{0,3}-x_{5} B_{0,3}\right), \\
& M_{24}^{0}=\frac{1}{m}\left(x_{2} A_{0,4}-x_{5} B_{0,4}\right), \\
& M_{25}^{0}=\frac{1}{m}\left(x_{2} A_{0,5}-x_{5} B_{0,5}-B_{0}\right) \text {, } \\
& M_{26}^{0}=\frac{1}{m}\left(x_{2} A_{0,6}-x_{5} B_{0,6}\right), \\
& M_{31}^{0}=\frac{1}{m}\left(x_{3} A_{0,1}-x_{6} B_{0,1}\right), \\
& M_{32}^{0}=\frac{1}{m}\left(x_{3} A_{0,2}-x_{6} B_{0,2}\right), \\
& M_{33}^{0}=\frac{1}{m}\left(x_{3} A_{0,3}+A_{0}-x_{6} B_{0,3}\right) \text {, } \\
& M_{34}^{0}=\frac{1}{m}\left(x_{3} A_{0,4}-x_{6} B_{0,4}\right), \\
& M_{35}^{0}=\frac{1}{m}\left(x_{3} A_{0,5}-x_{6} B_{0,5}\right), \\
& M_{36}^{0}=\frac{1}{m}\left(x_{3} A_{0,6}-x_{6} B_{0,6}-B_{0}\right) \text {, } \\
& M_{41}^{0}=\frac{1}{\mu}\left(x_{4} C_{0,1}-x_{1} B_{0,1}-B_{0}\right), \\
& M_{42}^{0}=\frac{1}{\mu}\left(x_{4} C_{0,2}-x_{1} B_{0,2}\right), \\
& M_{43}^{0}=\frac{1}{\mu}\left(x_{4} C_{0,3}-x_{1} B_{0,3}\right), \\
& M_{44}^{0}=\frac{1}{\mu}\left(x_{4} C_{0,4}+C_{0}-x_{1} B_{0,4}\right) \text {, } \\
& M_{45}^{0}=\frac{1}{\mu}\left(x_{4} C_{0,5}-x_{1} B_{0,5}\right), \\
& M_{46}^{0}=\frac{1}{\mu}\left(x_{4} C_{0,6}-x_{1} B_{0,6}\right), \\
& M_{51}^{0}=\frac{1}{\mu}\left(x_{5} C_{0,1}-x_{2} B_{0,1}\right), \\
& M_{52}^{0}=\frac{1}{\mu}\left(x_{5} C_{0,2}-x_{2} B_{0,2}-B_{0}\right),
\end{aligned}
$$




$$
\begin{gathered}
M_{53}^{0}=\frac{1}{\mu}\left(x_{5} C_{0,3}-x_{2} B_{0,3}\right), \\
M_{54}^{0}=\frac{1}{\mu}\left(x_{5} C_{0,4}-x_{2} B_{0,4}\right), \\
M_{55}^{0}=\frac{1}{\mu}\left(x_{5} C_{0,5}+C_{0}-x_{2} B_{0,5}\right), \\
M_{56}^{0}=\frac{1}{\mu}\left(x_{5} C_{0,6}-x_{2} B_{0,6}\right), \\
M_{61}^{0}=\frac{1}{\mu}\left(x_{6} C_{0,1}-x_{3} B_{0,1}\right), \\
M_{62}^{0}=\frac{1}{\mu}\left(x_{6} C_{0,2}-x_{3} B_{0,2}\right), \\
M_{63}^{0}=\frac{1}{\mu}\left(x_{6} C_{0,3}-x_{3} B_{0,3}-B_{0}\right), \\
M_{64}^{0}=\frac{1}{\mu}\left(x_{6} C_{0,4}-x_{3} B_{0,4}\right), \\
M_{65}^{0}=\frac{1}{\mu}\left(x_{6} C_{0,5}-x_{3} B_{0,5}\right), \\
M_{66}^{0}=\frac{1}{\mu}\left(x_{6} C_{0,6}+C_{0}-x_{3} B_{0,6}\right) .
\end{gathered}
$$

[1] H. Poincaré, Acta Mathematica 13, 1 (1890); Bull. Astronomique 8, 12 (1891).

[2] H. Poincaré, Les Methodes Nouvelles de la Mecanique Celeste (Gauthier-Villars, Paris, 1892), reprinted as New Methods of Celestial Mechanics, edited by D. L. Goroff (American Institute of Physics, 1993).

[3] J. F. Donoghue, Phys. Rev. Lett. 72, 2996 (1994).

[4] J. F. Donoghue, Phys. Rev. D 50, 3874 (1994).

[5] J. F. Donoghue, General relativity as an effective field theory: the leading quantum corrections (gr-qc/9405057).

[6] I. J. Muzinich and S. Vokos, Phys. Rev. D 52, 3472 (1995).

[7] H. W. Hamber and S. Liu, Phys. Lett. B 357, 51 (1995).

[8] A. A. Akhundov, S. Bellucci, and A. Shiekh, Phys. Lett. B 395, 16 (1997).

[9] I. B. Khriplovich and G. G. Kirilin, Sov. Phys. JETP 95, 981 (2002).

[10] N. E. J. Bjerrum-Bohr, J. F. Donoghue, and B. R. Holstein, Phys. Rev. D 67, 084033 (2003). 
[11] E. Battista and G. Esposito, Phys. Rev. D 89, 084030 (2014).

[12] L. A. Pars, A Treatise on Analytical Dynamics (Heinemann, London, 1965).

[13] G. Huang and X. Wu, Phys. Rev. D 89, 124034 (2014).

[14] A. Chenciner and R. Montgomery, Ann. Math. 152, 881 (2000).

[15] A. F. B. A. Prado and R. Broucke, Journal of Guidance, Control and Dynamics 19, 929 (1996).

[16] G. Gomez, Dynamics and Mission Design Near Libration Points: The Case of Collinear Libration Points. Fundamentals. Vol. I, World Scientific Monograph Series in Mathematics Vol. 2 (World Scientific, Singapore, 2001).

[17] J. Simo and C. R. McInnes, Solar sail trajectories at the Earth-Moon Lagrange points, in 59th Int. Astronomical Congress, Glasgow, Scotland (2008). 